\title{
Prinzmetal-Angina nach Lakritz-Konsum
}

Kathrin Machalke, Peter Bramlage, Karin Bramlage, Ulrich Tebbe

Lakritze wird aus Süßholzwurzel (Glycyrrhiza glabra) gewonnen, einer seit langem bekannten Heilpflanze. Wie man inzwischen weiß, kann chronisch erhöhter Lakritz-Verzehr zu Hypokaliämie und Hypertonie führen. Weniger bekannt sind Zusammenhänge mit einer vasospastischen Reaktion - neue Einblicke gibt der vorliegende Fallbericht.

\section{Erstes Ereignis}

Thorakaler Vernichtungsschmerz | Die 44-jährige Patientin (Größe: $160 \mathrm{~cm}$, Gewicht $65 \mathrm{~kg}$ ) wacht morgens mit thorakalem Vernichtungsschmerz und akuter Dyspnoe auf und alarmiert den Notarzt. Dieser stellt ein Hyperventilationssyndrom fest, der Blutdruck beträgt $90 / 65 \mathrm{mmHg}$, die Herzfrequenz 68 Schläge/min. Fünf Jahre vor dem aktuellen Ereignis kam es während einer Schwangerschaft zu einer tiefen Beinvenenthrombose; in der Folge stellte man eine heterozygote Prothrombinmutation fest. Medikamente nimmt die Frau derzeit nicht ein.

Erhöhtes Troponin I Nach Ankunft in der Klinik werden ein EKG und eine transthorakale Echokardiographie durchgeführt - beides ohne Auffälligkeiten; insbesondere findet sich keine Zeichen einer Lungenarterienthrombose. Die D-Dimere sowie der Kaliumspiegel liegen im Normbereich; der Troponinwert ist mit 0,041 und im Folgenden mit 0,955 $\mu \mathrm{g} / \mathrm{l}($ Norm $<0,003 \mu \mathrm{g} / \mathrm{l})$ erhöht.

Abb. 1 Links: Deutliche ST-Hebung in V2-V6 bei stationärer Aufnahme. Mitte: Nach 6 Stunden ist die ST- Hebung verschwunden; positive T-Welle in V2-V6. Rechts: Am Folgetag zeigen sich negative T-Wellen in V1-V4.

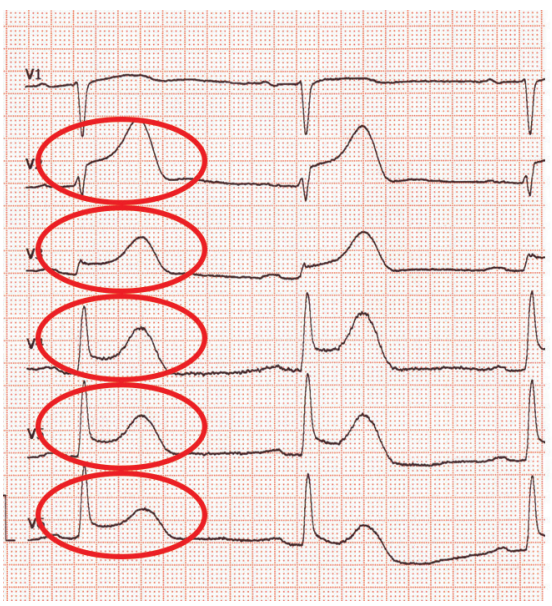

Linksherzkatheterisierung unauffällig | Die Beschwerden halten - etwas abgeschwächt - an, so dass am Nachmittag eine Linksherzkatheteruntersuchung erfolgt: Dabei kann eine stenosierende koronare Herzkrankheit ausgeschlossen werden. Auch ein Röntgen-Thorax in 2 Ebenen, ein Kardio-MRT sowie eine Bodyplethysmographie bleiben unauffällig. Die Schmerzen sistieren im weiteren Verlauf, und die Patientin kann entlassen werden. Eine spezifische Therapie wird nicht empfohlen.

\section{Zweites Ereignis}

Erneute thorakale Beschwerden | Vier Monate später treten morgens kurz nach dem Aufstehen - aus völligem Wohlbefinden heraus - erneut thorakale Beschwerden mit Dyspnoe auf. Die Patientin kommt zur Notaufnahme. Es stellt sich heraus, dass sie am Vortag - entgegen ihrer Gewohnheit - eine Tüte Lakritze ( $200 \mathrm{~g}$ ) gegessen hatte. Der Blutdruck beträgt 110/50 mmHg. Zum Aufnahmezeitpunkt ist der Troponinwert unauffällig $(<0,010 \mu \mathrm{g} / 1)$, jedoch bei einer Kontrolle am späten Nachmittag mit 0,225 $\mu \mathrm{g} / 1$ erhöht. DDimere, Kreatinkinase (CK) sowie CK-MB und Kaliumspiegel liegen im Normbereich, ebenso das Brain Natriuretic Peptide (BNP).

Gefäßerweiterung nach Nitroglycerin I Im Aufnahme-EKG zeigen sich Infarkt-typische STHebungen in den Ableitungen V2-V6 ( Abb. 1, links), sodass umgehend eine erneute Koronarangiografie erfolgt. Die Koronarien weisen keine Stenosen oder Embolien auf ( $\triangleright$ Abb. 2). Die LV-

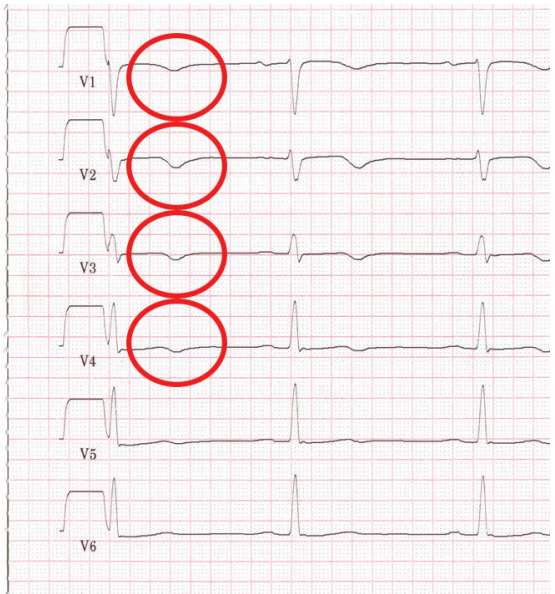


Funktion ist regional leicht eingeschränkt mit einer anteroapikalen Hypokinesie. Nach intrakoronarer Nitrogabe zeigte sich eine deutliche Gefäßerweiterung, was den Verdacht auf einen Gefäßspasmus bestätigt. Sechs Stunden nach der Aufnahme finden sich im EKG keine ST-Hebungen mehr $(\checkmark$ Abb. 1, Mitte). Auch am Folgetag fehlen ST-Hebungen; dafür finden sich T-Negativitäten in V1-V4 ( Abb. 1, rechts). Diese Befunde sprechen für eine Prinzmetal- Angina.

Therapie I Wir raten der Patientin, in Zukunft auf Lakritze zu verzichten, und verordnen $2,5 \mathrm{mg}$ Amlodipin/Tag. Seitdem sind ähnliche Beschwerden ausgeblieben.

\section{Diskussion}

Lakritze - ein Süßstoff | Lakritzprodukte enthalten mindestens 5\% eingedickten, trockenen Wasserextrakt der Süßholzwurzel (Süßholzsaft). Natürlicher Bestandteil ist Glycyrrhizin(säure) ( Abb. 3): Das Aglykon Glycyrrhetinsäure wird intestinal freigesetzt. Glycyrrhizin hat eine 50fach höhere Süßkraft als Rohrzucker, während das Aglykon nicht süß schmeckt. Medizinisch eingesetzt wird Lakritze als Expektorans bei Katarrhen der oberen Atemwege, als Antiphlogistikum, als Spasmolytikum bei Gastritis und Magengeschwüren sowie als Geschmackskorrigens [5].

Beschränkter Konsum empfohlen | Der Gehalt an Glycyrrhizin beträgt in den meisten Lakritzwaren maximal $200 \mathrm{mg} / 100 \mathrm{~g}$. Produkte mit einem höheren Gehalt müssen als Stark-Lakritz gekennzeichnet werden. Übersteigt der Gehalt $400 \mathrm{mg} / 100 \mathrm{~g}$, so muss folgender Hinweis angebracht sein: „Enthält Lakritze - bei hohem Blutdruck sollte ein übermäßiger Verzehr dieses Erzeugnisses vermieden werden“ [3]. Das Bundesinstitut für Risikobewertung empfiehlt den Konsumenten derzeit, die Glycyrrhizin-Zufuhr bei regelmäßigem Konsum auf $100 \mathrm{mg}$ pro Tag zu beschränken [4]. Die meisten Lakritzprodukte enthalten deutlich weniger als die sehr restriktiv definierte Grenze. Man erreicht diesen Wert erst bei einem Konsum von ca. 50-100 g Lakritze pro Tag. Unsere Patientin konsumierte allerdings $200 \mathrm{~g}$.

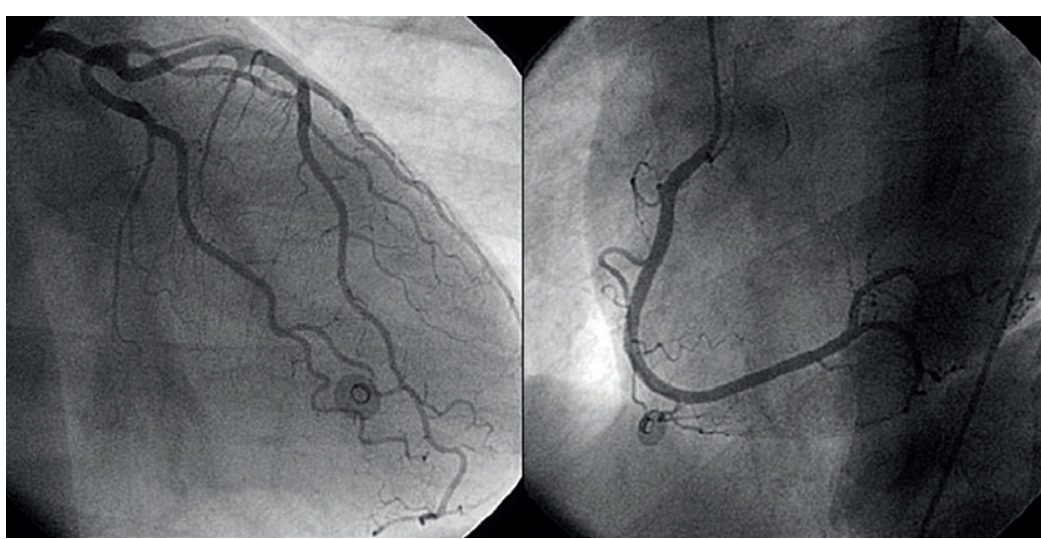

Abb. 2 Die Koronarangiografie der linken und rechten Koronararterie (LCA) zeigt einen

Mineralokortikoide Wirkung | Glycyrrhetinsäure hat zusätzlich mineralokortikoide Wirkung. Sie hemmt die 11-Beta-Hydroxysteroid-Dehydrogenase 2 (11-BHSD2), die Cortisol zu Cortison inaktiviert $($ Abb. 4). Dadurch kommt es zu einer dauerhaften Aktivierung des Mineralokortikoidrezeptors (MR) durch Cortisol.

- Die Affinität von Cortisol zum Rezeptor entspricht zwar derjenigen von Aldosteron,

- die Cortisol-Plasmakonzentration übersteigt die von Aldosteron jedoch um das Hundertfache.

Dadurch wird ein hyperaldosteroner Status wird vorgetäuscht [6]:

- Es kommt zu Änderungen im Mineralstoffhaushalt mit Natriumretention und Kaliumverlust.

- In der Folge steigt der Blutdruck, und es bilden sich Ödeme.

Freisetzung im Gefäßendothel | Das durch Glycyrrhetinsäure gehemmte Enzym 11-BHSD2 wird im distalen Tubulus der Niere, aber auch in der glatten Gefäßmuskulatur sowie in Endothelzellen

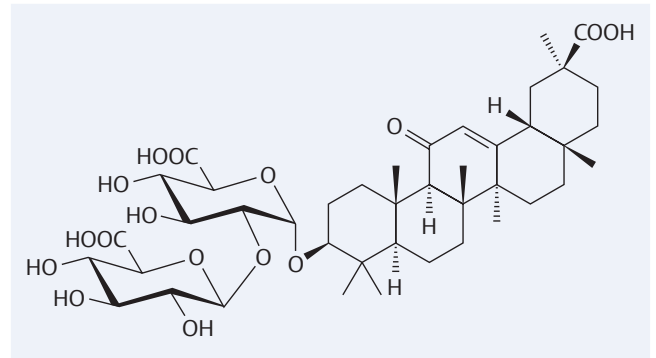

Abb. 3 Strukturformel von Glycyrrhizin.<smiles>CC(=O)OC(C)=O</smiles>

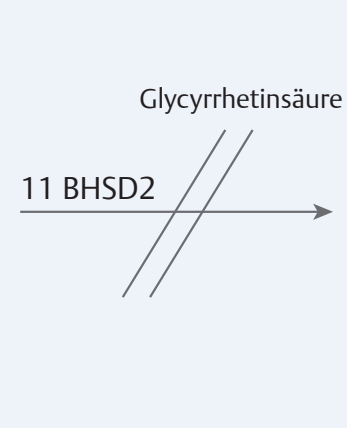

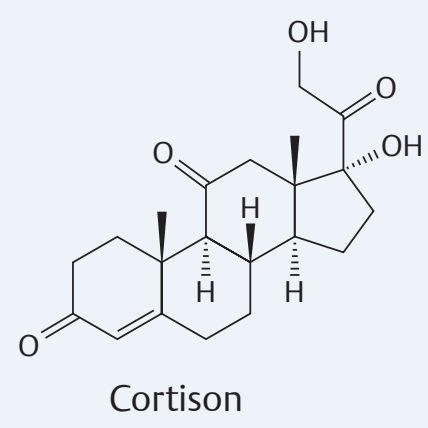

Abb. 4 Glycyrrhetinsäure hemmt die 11-Beta-Hydroxysteroid-Dehydrogenase 2 (11-BHSD2) und verhindert so die Inaktivierung von Cortisol zu Cortison. Das vermehrt anfallende Cortisol bindet an den Mineralokortikoid-Rezeptor; ein hyperaldosteroner Status wird vorgetäuscht. 


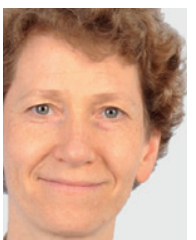

Dr. med. Kathrin Machalke ist Funktionsoberärztin an der Klinik für Kardiologie, Angiologie und internistische Intensivmedizin

Klinikum Lippe GmbH kathrin.machalke@klinikumlippe.de

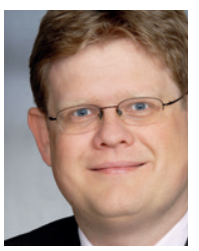

Prof. Dr. med Peter Bramlage ist Partner und geschäftsführender Gesellschafter des Instituts für Pharmakologie und präventive Medizin $\mathrm{GmbH}$, Mahlow

peter.bramlage@ippmed.de

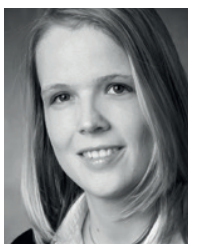

Dr. rer. medic Karin Bramlage ist wissenschaftliche Mitarbeiterin des Instituts für Pharmakologie und präventive Medizin GmbH, Mahlow karin.bramlage@ippmed.de

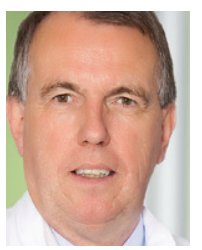

Prof. Dr. med. Ulrich Tebbe ist Chefarzt der Klinik für Kardiologie, Angiologie und internistische Intensivmedizin Klinikum Lippe GmbH ulrich.tebbe@klinikum-lippe.de

\section{Interessenkonflikt}

Die Autoren geben an, dass kein Interessenkonflikt besteht.

DOI 10.1055/s-0041-101294 Dtsch Med Wochenschr 2015; 140: 590-592

(C) Georg Thieme Verlag KG . Stuttgart · New York . ISSN 0012-0472 freigesetzt [7]. Es gibt Hinweise, dass der Verzehr von Lakritze die Vasokonstriktion der glatten Gefäßmuskulatur durch Hemmung der 11-BHSD2 mit späterer Veränderung des Endothelin-1- (Et-1) und Stickstoffmonoxid (NO)-Systems steigert [8].

Vasospasmen durch Lakritze | Vereinzelt wurde Lakritz-Konsum in Zusammenhang gebracht mit vasospastisch-vermitteltem vorübergehendem Sehverlust, Migräne und dem Posterioren reversiblen Enzephalopathie-Syndrom (PRES) [1, 2]. Zu den vasospastisch vermittelten Reaktionen gehören auch Spasmen der Koronargefäße, für die durch Glycyrrhizin oder Glycyrrhetinsäure induzierte Veränderungen in den Endothelin- und NO-Systemen verantwortlich gemacht werden.

Prinzmetal-Angina | Bei der Prinzmetal-Angina führen Spasmen der Herzkrankgefäße zu einer passageren Ischämie der Herzmuskulatur. Im Gegensatz zur klassischen Angina pectoris treten die Beschwerden belastungsunabhängig - häufig früh morgens - auf, begleitet von retrosternalem Druck bzw. Schmerz, der in Nacken, Kiefer und/oder die linke Schulter ausstrahlen kann. Mögliche Begleiterscheinungen sind Palpitationen und Synkopen verursacht durch Arrhythmien. Schlimmstenfalls kann ein Herzinfarkt resultieren. Betroffen sind vor allem junge Menschen im Alter von 30-40 Jahren, häufig Raucher; nur selten liegen kardiovaskuläre Risikofaktoren vor. Im EKG kann während eines Anfalls eine ST-Strecken-Hebung dargestellt werden. Differenzialdiagnostisch muss zwischen der Prinzmetal-Angina und einer instabilen Angina pectoris, bedingt durch eine Atherosklerose der Koronargefäße, unterschieden werden. Aufschluss gibt hier eine Koronarangiografie.

Mögliche Wirkmechanismen | Erkenntnisse zu pathophysiologischen Zusammenhängen stammen überwiegend aus Tierexperimenten:

- Parisella et al. [9] untersuchten den Effekt von Glycyrrhizin und Glycyrrhetinsäure an isolierten Rattenherzen. Sie stellten fest, dass beide Substanzen direkt die kardiale Kontraktilität und Erschlaffung beeinflussen konnten - mit zum Teil gegenteiligen Effekten.

- Im Aortengewebe von Ratten mit Glycyrrhizinsäure-induzierter Hypertonie fanden Ruschitzka et al. [10] erhöhte Endothelin-1- und verringerte eNOS- sowie Nitratkonzentrationen. Nach Gabe von MR-Antagonisten (Spironolacton, Epleneron) sowie unter ETA-Blockade normalisierten sich der Blutdruck und die Veränderungen der ET-1-, eNOS- und Nitratwerte. Durch chronische Lakritz-Zufuhr wurden ETA-induzierte Kontraktionen der Aortenringe verstärkt. Das ließ sich sowohl durch Gabe von Aldosteron-Rezeptor-Antagonisten als auch durch eine ETABlockade bessern.

- Eine Beeinträchtigung der Gefäßentspannung als Antwort auf eine Acetylcholin-Gabe an zuvor verengten renalen Arterienringen von hy- pertensiven Ratten (Lakritze-induziert) konnte durch Einsatz von Spironolacton komplett normalisiert und unter dem ETA-Rezeptor-Antagonist Darusentan lediglich verbessert werden. Nitroprussid beeinflusste die Endothel-unabhängige Erschlaffung nicht [11].

- Sobieszczak et al. [12] kamen in ihren Untersuchungen zu dem Schluss, dass eine durch Glycyrrhetinsäure-vermittelte Hemmung von 11-BHSD2 die gefäßerweiternde Funktion der Gefäßmuskulatur abschwächt.

Genetisch bedingt höhere Sensibilität? | Miettinen et al. [13] untersuchten die DNA-Proben von 30 Probanden mit dokumentierter Lakritze-induzierter Hypertonie. Sie konnten keine signifikanten DNA-Variationen der 11-BHDS2-Gene identifizieren. Allerdings waren DNA-Varianten der Untereinheiten des epithelialen Natriumkanals, die mit einer Hypertonie in Verbindung stehen sollen, bei den Studienteilnehmer mit 20\% häufiger als bei einer Vergleichsgruppe von Blutspendern (3,7\%). Demnach - so folgerten die Autoren - könnten Varianten der ENaC-Untereinheiten einige Individuen für eine durch Lakritze ausgelöste Hypertonie sensibler machen.

Differenzialdiagnose | Es gibt auch Berichte über allergische Reaktionen durch Lakritze, z.B. Hautausschläge oder Berufsasthma [13, 14]. Zu allergischen Koronarspasmen durch Lakritze fehlen jedoch Literatur-Daten. Hier kann man das KounisSyndrom als allergischen Myokardinfarkt anführen [15]. Man unterscheidet bei diesem Syndrom zwei Typen. Beim Typ I liegen morphologisch unauffällige Koronargefäße vor. Durch eine allergische Reaktion kommt es über eine endotheliale Dysfuktion infolge eines Vasospasmus zu Angina pectoris oder zum Myokardinfarkt. Eine Koronarsklerose liegt beim Typ II zugrunde. Durch eine allergische Reaktion kommt es zu Plaqueerosion oder - ruptur und dann zur Thrombusbildung und zum Myokardinfarkt [16].

\section{Konsequenz für Klinik und Praxis}

- Lakritzprodukte sind als „low-fat“ Genusswaren weit verbreitet - dass der vermehrte Konsum schädlich sein kann, ist vielen Konsumenten unbekannt.

- Im geschilderten Fall kam es zu einem Koronarspasmus (Prinzmetal-Angina), bei dem aufgrund der bereits erforschten Einflüsse von Glycyrrhizin und Glycyrrhetinsäure auf das Endothelin- bzw. NO-System eine Assoziation mit einem Lakritz-Konsum denkbar ist.

\section{Literatur}

1 Dobbins KR, Saul RF. Transient visual loss after licorice ingestion. J Neuro-ophthalm 2000; 20: 38-41 
2 Morgan RD, Chou SH, Stelfox HT. Posterior reversible encephalopathy syndrome in a patient following binge liquorice ingestion. J Neurol 2011; 258: 1720-1722

3 Richtlinie 2004/77/EG der Kommission vom 29. April 2004 zur Änderung der Richtlinie 94/54/EG hinsichtlich der Kennzeichnung bestimmter Lebensmittel, die Glycyrrhizinsäure und deren Ammoniumsalz enthalten. http://eur-lex.europa.eu/LexUriServ/LexUriServ. do?uri=OJ: L: 2004: 162: 0076: 0077: DE: PDF (letzter Zugriff 17. 03. 2015)

4 Bundesinstitut für Risikobewertung. Lakritzkonsum und Glycyrrhizin. http://www.bfr.bund.de/de/presseinformation/1999/02/bgvv_raet_zur_vorsicht_beim_verzehr_von_lakritze_-861.html (letzter Zugriff 17. 03. 2015)

5 Wichtl M. Teedrogen und Phytopharmaka. Stuttgart: Wissenschaftliche Verlagsgesellschaft mbH; 1997

6 Mutschler EG, Geisslinger G, Kroemer HK, SchäferKorting M. Mutschler Arzneimittelwirkungen. Stuttgart: Wissenschaftliche Verlagsgesellschaft; 2001

7 Walker BR. Glucocorticoids and cardiovascular disease. Eur J Endocrinol 2007; 157: 545-559

8 Konik E, Kurtz EG, Sam F et al. Coronary artery spasm, hypertension, hypokalemia and licorice. J Clin Case Rep 2012; $2: 1-3$

9 Parisella ML, Angelone T, Gattuso A et al. Glycyrrhizin and glycyrrhetinic acid directly modulate rat cardiac performance. J Nutr Biochem 2012; 23: 69-75
10 Ruschitzka F, Quaschning T, Noll G et al. Endothelin 1 type a receptor antagonism prevents vascular dysfunction and hypertension induced by 11 betahydroxysteroid dehydrogenase inhibition: role of nitric oxide. Circulation 2001; 103: 3129-3135

11 Quaschning T, Ruschitzka F, Niggli B et al. Influence of aldosterone vs. endothelin receptor antagonism on renovascular function in liquorice-induced hypertension. Nephrol Dialys Transplant 2001; 16: 2146-2151

12 Sobieszczyk P, Borlaug BA, Gornik HL et al. Glycyrrhetinic acid attenuates vascular smooth muscle vasodilatory function in healthy humans. Clin Sci 2010; 119: 437-442

13 Benomar S, Ismaili N, Koufane ] et al. [Fixed food eruption caused by liquorice]. Ann Derm Vénérol 2010; 137: $121-123$

14 Cartier A, Malo JL, Labrecque M. Occupational asthma due to liquorice roots. Allergy 2002; 57: 863

15 Kounis NG, Mazarakis A, Tsigkas G et al. Kounis syndrome: a new twist on an old disease. Future Cardiol 2011; 7: 805-824

16 Kraus J. Der allergische Myokardinfarkt - Kounis-Syndrom. J Kardiol 2012; 19 : 118-122 